\title{
Re: Treatment decisions and outcome in very elderly patients with diffuse large B-cell lymphoma
}

\author{
Jessica N. Williams, MD MPH ${ }^{1}$, Jean L. Koff, MD², and Christopher R. Flowers, MD MS ${ }^{2}$ \\ ${ }^{1}$ Emory University School of Medicine, Atlanta, GA \\ ${ }^{2}$ Winship Cancer Institute, Emory University, Atlanta, GA
}

To the Editor,

We appreciate the insightful letter by Fabbri et al regarding our manuscript "Disease Characteristics, Patterns of Care, and Survival in Very Elderly Patients with Diffuse Large B-Cell Lymphoma ${ }^{1}$." As patients $>80$ years old have the highest incidence of diffuse large B-cell lymphoma (DLBCL) yet are rarely included in studies, we sought to characterize treatment and survival patterns in this population. We found that in DLBCL patients $>80$ years $(n=1,156)$, rituximab, cyclophosphamide, doxorubicin, vincristine, and prednisone ( $R$ $\mathrm{CHOP}$ ) was associated with the longest survival, even after controlling for potential confounders such as performance status and comorbidity.

Although our data suggest that age alone should not be a contraindication to effective treatment, we agree with Fabbri et al that anthracycline toxicity is a major concern in very elderly patients given their decreased functional and physiologic reserve. Indeed, a recent study found that approximately one-third of elderly DLBCL patients who received anthracycline-based therapy experienced toxicity requiring treatment modification ${ }^{2}$. As alternatives, Fabbri et al thoughtfully suggest reduced doses of R-CHOP, non-anthracyclinebased regimens, and non-pegylated liposomal doxorubicin in this population.

In addition to the aforementioned treatment modifications, radiation therapy may also be beneficial for elderly DLBCL patients. A recent study revealed that elderly patients with limited stage DLBCL treated with abbreviated R-CHOP and radiation $(n=359)$ had a decreased risk of second-line therapy and febrile neutropenia as compared to patients treated with full-course R-CHOP alone ( $\mathrm{n}=515)$; overall survival was similar between the two groups $^{3}$. These results suggest that abbreviated R-CHOP with radiation may be better tolerated in elderly patients with limited stage DLBCL, and future studies should further investigate this treatment regimen. In conclusion, we agree with the points raised by Fabbri et al and advocate for further studies examining alternative DLBCL treatment regimens for elderly patients who cannot tolerate standard R-CHOP.

Corresponding Author: Christopher R. Flowers MD, MS, Associate Professor, Department of Hematology and Oncology, 1365 Clifton Road N.E., Building B, Suite 4302, Emory University, Atlanta, GA 30322, Phone: 404-778-5554, Fax: 404-778-3366, crflowe@emory.edu.

Conflicts of Interest: There are no relevant conflicts of interest to disclose. 


\section{Acknowledgments}

This work was supported by Dr. Flowers' National Cancer Institute R21 CA158686 and Dr. Nastoupil's American Society of Hematology Clinical Scholars Award.

\section{References}

1. Williams JN, Rai A, Lipscomb J, Koff JL, Nastoupil LJ, Flowers CR. Disease characteristics, patterns of care, and survival in very elderly patients with diffuse large B-cell lymphoma. Cancer. published online February 11, 2015. 10.1002/cncr.29290

2. Davis CC, Cohen JB, Shah KS, et al. Efficacy and tolerability of anthracycline-based therapy in elderly patients with diffuse large B-cell lymphoma. Clin Lymphoma Myeloma Leuk. 2015; 15:270-277. [PubMed: 25704479]

3. Odejide OO, Cronin AM, Davidoff AJ, LaCasce AS, Abel GA. Limited stage diffuse large B-cell lymphoma: comparative effectiveness of treatment strategies in a large cohort of elderly patients. Leuk Lymphoma. 2015; 56:716-724. [PubMed: 24913508] 